\title{
Metabolite characterisation and profiling of Hermetia illucens L. larvae at various growth stages using Sesamum indicum residues as nutrient source
}

\author{
S.-W. Hsiao ${ }^{1 \#}$, I-C. Kuo ${ }^{2 \#}$, C.-W. Su ${ }^{3}$, Y.-H. Wang ${ }^{4}$, H.-C. Mei ${ }^{5^{*}}$ and C.-K. Lee ${ }^{1,3,4^{*}}$ \\ ${ }^{1}$ Ph.D. Program in Drug Discovery and Development Industry, College of Pharmacy, Taipei Medical University, Taipei 11031, \\ Taiwan; ${ }^{2}$ Department of Microbiology and Immunology, University of British Columbia, Vancouver, BC V6T 1Z3, Canada; \\ ${ }^{3}$ Graduate Institute of Pharmacognosy, Taipei Medical University, Taipei 11031, Taiwan; ${ }^{4}$ School of Pharmacy, Taipei \\ Medical University, Taipei 11031, Taiwan; ${ }^{5}$ Department of Science Education, National Taipei University of Education, \\ Taipei 10671, Taiwan; hcmei@tea.ntue.edu.tw; cklee@tmu.edu.tw; "these authors contributed equally to this work
}

Received: 7 March 2021 / Accepted: 21 June 2021

(c) 2021 Wageningen Academic Publishers

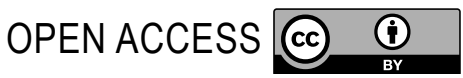

RESEARCH ARTICLE

\begin{abstract}
The larvae of black soldier fly (Hermetia illucens L.) (BSFL) is an excellent decomposer for industrial and agricultural applications capable of converting low-value waste into nutritional biomass. Sesame residues is industrial food waste possess high protein and lipid content. To investigate the bioconversion process of BSFL, the larvae were raised on a low-bioburden diet consisting of sesame residue, and liquid chromatography-mass spectrometry (LC-MS) analysis was performed on the BSFL and faeces at various instars. The obtained metabolomes were subsequently evaluated through principal component analysis and orthogonal projection to latent structure-discriminant analysis to explore the differential components. Diacylglycerol was the main component of the sesame residues utilised by BSFL during metabolism. The phenylalanine, arginine, oleic acid and 10-hydroxy-8E-octadecenoic acid were identified as the major components in various instars of the BSFL metabolites. We thus concluded that BSFL have different nutritional compositions at different instar stages. Another compound with a molecular weight of 278.224 prevalent in both the larvae and faeces might play vital roles in BSFL development. The present study revealed a part of metabolic changes important in the BSFL lifecycle. Through this pioneering metabolomics profiles, the discovery of highly-valuable biomolecules on BSFL for food industrial application can be anticipated.
\end{abstract}

Keywords: black soldier fly, sesame, metabolomics, amino acids, fatty acids

\section{Introduction}

According to WHO, the world population has exceeded 7 billion, with 87 million people living in poverty and starvation. The global population is likely to reach 9 billion by 2050 , and the amount of food produced needs to be at least doubled to fulfil the demand (Alexandratos and Bruinsma, 2012). Human beings are inevitably forced to face challenges such as land and water shortages, overfishing and climate changes (McKee, 2003). In the long run, famine is undoubtedly a growing crisis.

Food and Agriculture Organization of the United Nations declared that edible insects provide the future prospects for food and feed security (Van Huis et al., 2013). There are more than 1900 types of edible insects which serve as the staple food for at least 2 billion people. From the nutritional aspects of human consumption, edible insects are highly nutritious that provide dietary energy, proteins, fatty acids, fibres, dietary minerals (Mwangi et al., 2018) and vitamins (Van Huis et al., 2013). Along with the abundance and diversity of insects on earth makes entomophagy the ideal solution for alleviating the global food crisis (ChurchwardVenne et al., 2017). One promising species that not only serves as a raw material for the chemical industry and is being industrially reared as an alternative protein source for animal feed is Hermetia illucens L., the black soldier fly (BSF) (De Smet et al., 2018; Spranghers et al., 2017). The amino acid composition and high digestible indispensable amino acid score $(>75 \%)$ values indicated that the BSF larvae 
(BSFL) proteins could be utilised as a good source of animal protein for human nutrition as well (Huang et al., 2019).

BSF usually colonise near decaying organic matter such as animal waste or plant material. In the larval stage, their ability in consuming decaying matter has been used to reduce animal manure in commercial swine and poultry facilities (Newton et al., 2005). The life cycle of BSF can be sequentially divided into eggs, larvae, pupae and adult stages. Before reaching pupation, the larvae undergo six instars and spend approximately 14 days to complete the larval development (Hall and Gerhardt, 2002). The sixth instar larvae will then migrate to dry sheltered areas where the exoskeleton darkens, and a pupa develops within. After about two weeks of pupation, an adult BSF can live for approximately 5-8 days (Hall and Gerhardt, 2002).

The BSFL, also known as the Phoenix Worm, is currently used in animal feed, organic fertiliser and protein supplement (Wang and Shelomi, 2017). Besides their use for the sustainable production of proteins and lipids (Müller et al., 2017), studies also showed BSFL has novel antibacterial peptides (Elhag et al., 2017) and bioactive substances which could potentially be used for human and animal welfare (Müller et al., 2017). Correspondingly, Park et al. indicated a novel antimicrobial peptide, defensin-like peptide 4, exhibiting activity against Gram-positive bacteria including methicillin-resistant Staphylococcus aureus was extracted and purified from the immunised haemolymph of BSFL (Park et al., 2015). In terms of black soldier fly's contribution to bioconversion technology, Jiang et al. found the BSFL gut microbiome serves as an engine for food waste bioconversion, which is conducive to bioproducts regeneration from waste (Jiang et al., 2019).

Sesamum indicum, commonly known as sesame, is an annual plant majorly distributing in tropical and subtropical areas. It can be divided into various species with most popular off-white and black appearances. Sesame is considered as a health-promoting ingredient not only because that its seeds contain 45-60\% lipid oil, most of which are unsaturated linoleic and linolenic acids, but also the residues enclose multiple nutritional components such as proteins, carbohydrates, fibres, vitamins, and minerals. In eastern consumption habits, sesame is widely used as edible oil, additives and for medicinal purpose. Shennong's Classic of Materia Medica stated that sesame improves hair quality, relieves constipation, hypertension, and detoxification. Various biological benefits of components in sesame are also extensively discussed: lignan compounds are natural antioxidants (Miraj and Kiani, 2016), triterpenoids have anti-inflammatory effects (Akihisa et al., 2000), fatty acids exhibit antibacterial abilities (Zhang et al., 2013).
Metabolomics is a newly developed science followed by genomics, transcriptomics and proteomics. The quantitative study of metabolomes reveals the physical status of the organisms as well as the conceivable ongoing biochemical pathway. In this paper, we comprehensively investigated the metabolome of BSFL at different growth stages fed on sesame residues to explore whether BSFL has the potential to become a key bioconversion player and valuable nutritional alternative.

\section{Materials and methods}

\section{Insect rearing and sample collection}

The $300 \mathrm{~g}$ of BSFL and the $25 \mathrm{~kg}$ of sesame (S. indicum seed) residues used for the purpose of this study were both provided by the farm from Raycome International Co. located in Taoyuan, Taiwan. The sesame residue, the waste during cold-pressed sesame oil production, was fermented for 15 days after adding 10 times the volume of water. The larvae were cultured in the laboratory (School of Pharmacy, Taipei Medical University) under controlled temperature, humidity and light cycle $\left(26-29^{\circ} \mathrm{C}, 70 \%\right.$ r.h., $14: 10 \mathrm{~h}$ light: dark). At the start of rearing, about 900 larvae were reared in two plastic boxes $(34 \times 18 \times 7 \mathrm{~cm})$. Along with the growth of larvae, the remaining numbers of larvae were about 750 at the $4^{\text {th }}$ instar stage and 250 at the $5^{\text {th }}$ instar stage. The larvae were only fed with $100 \mathrm{~g}$ of sesame residues every day. The ratio of larvae number to gram of sesame residues was about 9:1 at the $2^{\text {nd }}$ instar, 7.5:1 at the $4^{\text {th }}$ instar, and 2.5:1 at the $5^{\text {th }}$ instar. Similarly, the sesame residues were stored at room temperature in the lab and was allocated as the BSFL diet every day to maintain larvae growth until sacrifice.

The larvae hatched and delivered from the farm were divided into four groups with one group treated by liquid nitrogen and sacrificed immediately as the $0^{\text {th }}$ stage control group (0b). The remaining larvae were fed with sesame residues every day, and 150-250 larvae were collected and sacrificed by liquid nitrogen after reaching the $2^{\text {nd }}(2 b), 4^{\text {th }}$ (4b) and $5^{\text {th }}(5 \mathrm{~b})$ instars respectively. The size of larvae was about $1 \mathrm{~cm}$ at the $2^{\text {nd }}$ instar, $1.6 \mathrm{~cm}$ at the $4^{\text {th }}$ instar, and $1.8 \mathrm{~cm}$ at the $5^{\text {th }}$ instar. The faeces from all three groups were also collected daily for analysis (labelled as 2, 4 and 5 $\mathrm{m})$. The faeces samples were collected directly by tweezers and separated from sesame residues as well as possible. Larvae go through six instars with the ecdysis marking the transition between each instar. Since moulting involves the shedding of both exoskeleton and intestinal epidermal layer, the larvae consume the intestinal part of the exuviae after ecdysis to re-establish the microbiota. As a result of this unique characteristic, the larvae and their faeces were sacrificed and collected seven days after moulting to ensure the completion of such process. 


\section{Extraction of sesame residue, larval and faecal metabolites}

Before the extraction, dd water was used to clean the surface of larvae. Ethanol ( $99.8 \%$, analytical reagent grade) was added to $0.5 \mathrm{~g}$ of sacrificed larvae and $3 \mathrm{~g}$ of faeces, and the samples were homogenised with TissueLyser II (Qiagen, Hilden, Germany) via sonication for 30 minutes. The supernatant from the centrifuged samples (Kubota Corporation, Tokyo, Japan) were filtered with a $0.22 \mu \mathrm{m}$ membrane and diluted to $1 \mathrm{mg} / \mathrm{ml}$ and $1 \mu \mathrm{g} / \mathrm{ml}$ concentrations in preparation for high-performance liquid chromatography with diode-array detection (HPLC-DAD) and LC-MS analyses respectively.

Likewise, extracts of sesame residues (ses) underwent the same processing steps to serve as controls for various metabolite analyses. $5 \mathrm{~g}$ of sesame residues was mixed with 10x equivalent volume of ethanol, sonicated for 30 minutes and centrifuged at 12,000 rpm for $5 \mathrm{~min}$ before the concentration was adjusted to $1 \mathrm{mg} / \mathrm{ml}$ and $1 \mu \mathrm{g} / \mathrm{ml}$ concentrations for HPLC-DAD and LC-MS analyses. The sampling of larvae and sesame residues was triplicated, and the sampling of faeces was duplicated.

\section{Extraction and purification of sesame residue}

The $8 \mathrm{~kg}$ of sesame residues was immersed in ethanol, extracted and vacuum-condensed after filtration. The ethanol extract (200 g) was subjected to silica gel (70230 mesh, Merck, Darmstadt, Germany) open column chromatography and eluted via a stepwise gradient method by using mixtures of $n$-hexane ( $n$-Hex), ethyl acetate (EA), methanol (Me) as the mobile phase. 70 fractions from various elution conditions were individually analysed by thin-layer chromatography (TLC) on silica gel 60 F254 plates (Merck). $n$-Hex-EA mixture was used for development, and spots were detected by spraying with vanillin-sulfuric acid followed by heating. Then, all fractions were grouped into 13 portions based on the similarity of composition of each fraction according to the results of the TLC analyses.

The semi-preparative HPLC was equipped with a Phenomenex Luna Silica (2) $100 \AA$ ( $250 \times 10 \mathrm{~mm})$ column and refractive index detector with a flow rate of $3 \mathrm{ml} / \mathrm{min}$. According to the result from HPLC-DAD analysis, the eluent from the open column chromatography containing the peak with the greatest difference in absorption peak as compared with BSFL samples was concentrated at the $23^{\text {rd }}$ fraction with a retention time (RT) of 26 mins (Figure 1). Henceforth, semi-preparative HPLC was performed on section 23 (S23) using n-Hex-EA (9:1) mixture as mobile phase for compound isolation and purification, and a total of 8 sub-fractions (S23-1 to S23-8) were isolated. Semipreparative HPLC was performed on sub-fraction S23-5 and S23-8, which were the major compounds contributing to the most absorption peak, using n-Hex-EA- $\mathrm{CH}_{2} \mathrm{Cl}_{2}$ (9:1:1) as mobile phase for further compound isolation and purification. The structure of the main purified substances was determined by ${ }^{1} \mathrm{H}$ and ${ }^{13} \mathrm{C}$-NMR spectroscopy.

\section{Analysis of metabolites in larvae and faeces}

The processed larvae metabolites and faeces were analysed using LC-MS. The flow rate was set at $1 \mathrm{ml} / \mathrm{min}$ for HPLC and $0.5 \mathrm{ml} / \mathrm{min}$ for UPLC. The column used for LC was the Thermo Syncronis C18 Column $(5 \mu \mathrm{m} 4.6 \times 250$ $\mathrm{mm})$. Experimental conditions and machine parameters established for analysis were outlined in Table S1 and S2.

The raw data from LC-MS was processed and automatically normalised using Progenesis QI v2.1 (Nonlinear Dynamics, Waters Corporation, UK). The dataset was imported into the software to conduct the retention time alignment, peak selection, and metabolite identification. The processed data consisting of molecular weight, retention time, signal intensity, molecular formula and molecular structure estimation based on database comparisons, was then exported to Microsoft Excel (Microsoft, Redmond, WA, USA). The organised excel data was subsequently imported into the SIMCA-P+ (Version 13) for multivariate analysis. Pareto scaling method was use for data scaling. The metabolite data and classification information were subjected to analysis using both unsupervised and supervised methods. A general overview of the main components among different samples and groups was obtained using the unsupervised principal component analysis (PCA). Subsequently, the supervised model, orthogonal projection to latent structure-discriminant analysis (OPLS-DA), was used to directly illustrate the differences in metabolites among groups through a scoring chart. The S-plot was used to do a preliminary selection on the biomarkers between two groups. Afterward, the relative abundance and its SD or SE value were calculated to determine the significance of these variables, as well as their likelihood to be biomarkers.

After processing the LC-MS data through software and statistical analysis, the ions showing significant differences between groups were listed as potential biomarkers. These potential biomarkers were analysed using tandem mass spectrometry to break down the precursor ions into fragments (product ions) to reveal the relevant chemical structure. The fragments revealed aspects of the chemical structure of the precursor ion that helped with profiling the metabolites and metabolic pathways involved in different developmental phases of BSFL. 


\section{Results}

\section{HPLC-DAD analysis of processed BSFL and S. indicum}

The extraction yield of crude extract (weight of extract/ weight of material $\times 100 \%$ ) of sesame residues, larvae, and faeces were $6.85 \pm 1.84,12.61 \pm 0.83$ and $1.20 \pm 0.61 \%$, respectively. The HPLC-DAD spectra (at $220 \mathrm{~nm}$ absorbance) of processed BSFL and $S$. indicum showed comparable UV absorbance patterns at 220, 250, 280 and $300 \mathrm{~nm}$. As illustrated in Figure 1, the ses and larvae harvested at different instar stages $(0 \mathrm{~b}, 2 \mathrm{~b}, 4 \mathrm{~b}, 5 \mathrm{~b})$ showed similar spectra patterns before the RT of $25 \mathrm{~min}$, while a sharp signal appeared in ses at RT of 26 min was absent in all the larval groups. The components extracted from the fraction with the RT of 26 min are one of the main focuses of our purification process.

\section{Compound isolation from S. indicum residue by semi- HPLC-DAD}

According to the inference made previously, the portion of the ses with the greatest difference in the absorption spectrum with RT of 26 min was concentrated in the $23^{\text {rd }}$ segment of fraction 2 (S23) (data not shown). Semipreparative HPLC confirmed that S23-5 and S23-8 contributed to the main absorbance peak with RT of 26 min. After repeated semi-HPLC separations, compounds 1 and 2 were confirmed to be the target components with the most obvious differences.

\section{Structural analysis of compound 1 and 2}

When ${ }^{1} \mathrm{H}$-NMR was used for structural analysis of unsaturated fatty acids, we discovered several chemical environments of protons, specifically: olefinic protons (5.3-5.4 ppm), protons attached to the bis-allylic carbons (2.7-2.8 ppm), protons attached to the allylic carbons (2.0$2.1 \mathrm{ppm})$ and the terminal methyl group protons (0.8-0.9 $\mathrm{ppm}$ ). These proton environments suggested the possibility for the compound to be unsaturated fatty acids.

According to the ${ }^{1} \mathrm{H}-\mathrm{NMR}$ spectrum of Compound 1 shown in Figure 2A, a chemical shift at the $\delta_{H}$ 5.2-5.4 location indicated the presence of olefinic protons. The peak at $\delta_{H}$ 2.7-2.8 represented bis-allylic protons where the protons were on a carbon that is between two sets of double bonded carbons. The $\delta_{H} 2.3$ shift was from protons in the $\mathrm{CH}_{2}$ located beside a ketone group. The series of peaks at $\delta_{H}$ 1.9-2.1 represented allylic protons where the methylene $\alpha$ olefin hydrogens of the $\mathrm{CH}_{2}$ group in the unsaturated fatty acid was connected to a double bond. The $\delta_{H} 1.2-1.3$ chemical shift represented protons of $\mathrm{CH}_{2}$ groups in a long hydrocarbon chain. The peaks located at $\delta_{H} 0.96$ presented protons from a $\mathrm{CH}_{3}$ located at the end of a $\omega$-3 unsaturated carbon chain, whereas the peaks at $\delta_{H} 0.87$ were signals from protons at the end of either a $\omega-6$ or $\omega-9$ methyl group. Chemical shifts at $\delta_{H} 4.1$ and $\delta_{H} 4.2$ on the other hand, came from the protons connected to a glycerol group. Based on the ${ }^{13} \mathrm{C}$-NMR spectrum of Compound 1 in Figure $2 \mathrm{~B}$, the chemical shifts at $\delta_{C} 65.3$ and $\delta_{C} 68.6$ revealed C-O bonding with approximately 2:1 ratio, whereas $\delta_{C} 174.1$ represented the presence of an ester group in this compound.

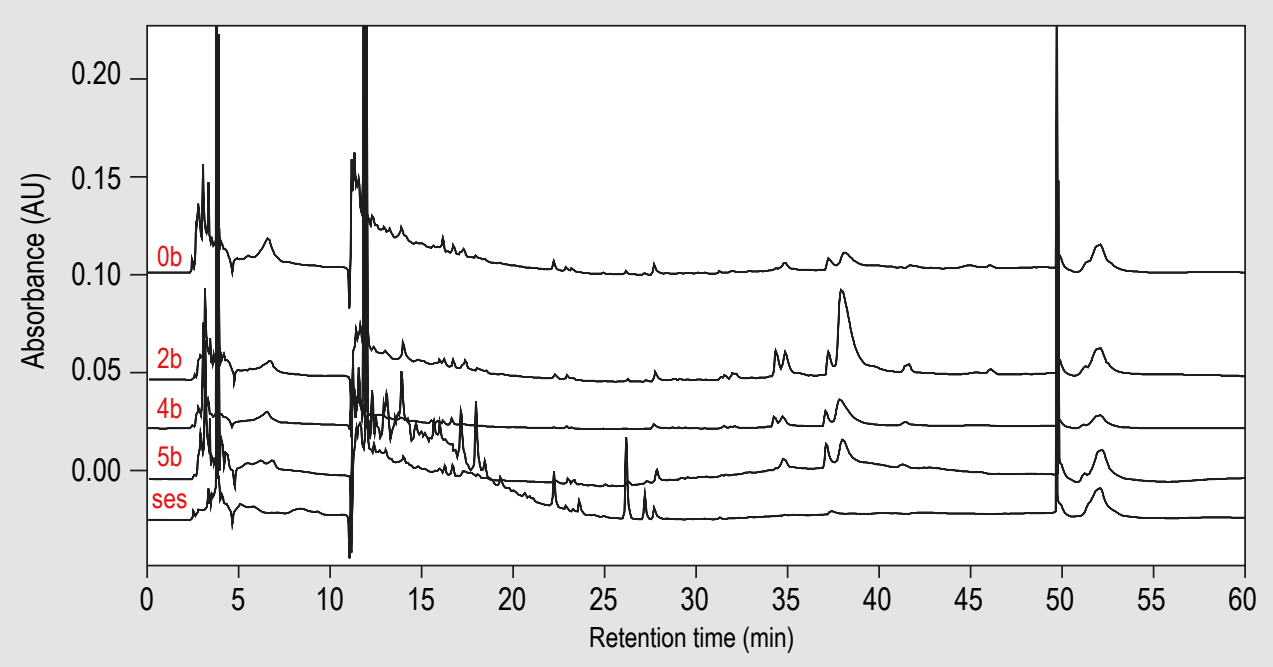

Figure 1. The chromatogram illustrating the absorbance (AU) patterns and retention times of compounds extracted from sesame (ses), and larvae metabolites at different growth stages (0b, 2b, $4 b$ and 5b). The compounds were separated using high-performance liquid chromatography using the diode-array detector method (HPLC-DAD) with the UV wavelength set at $220 \mathrm{~nm}$. 
A

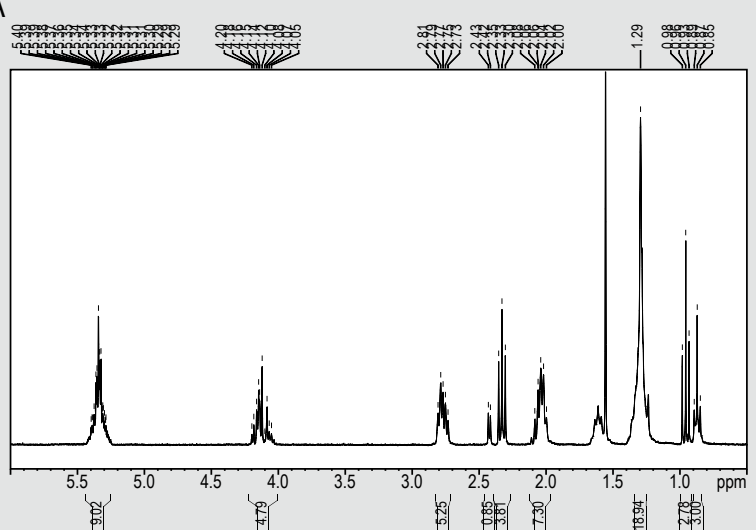

C

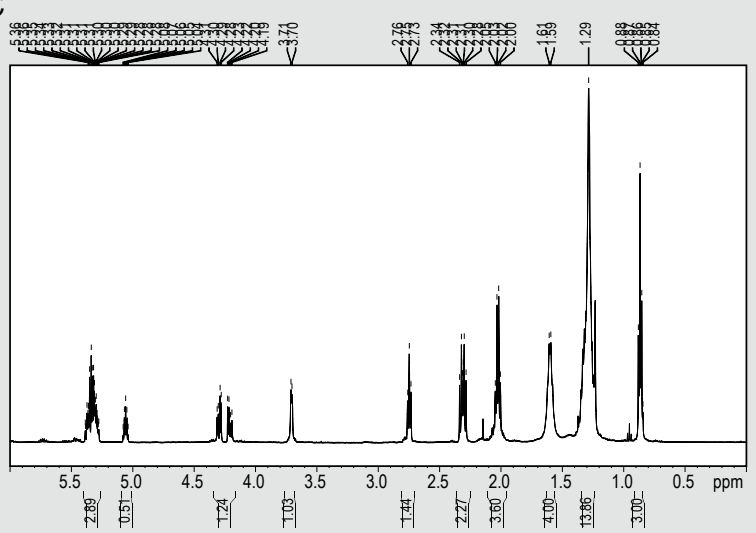

B

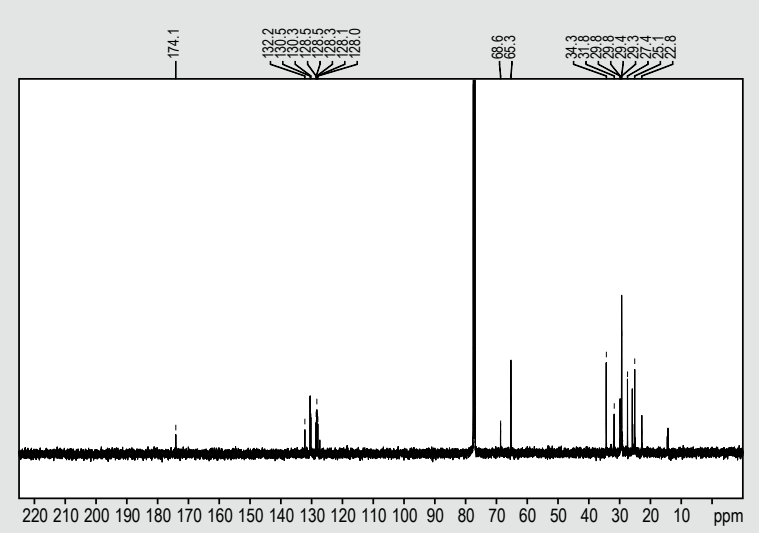

D

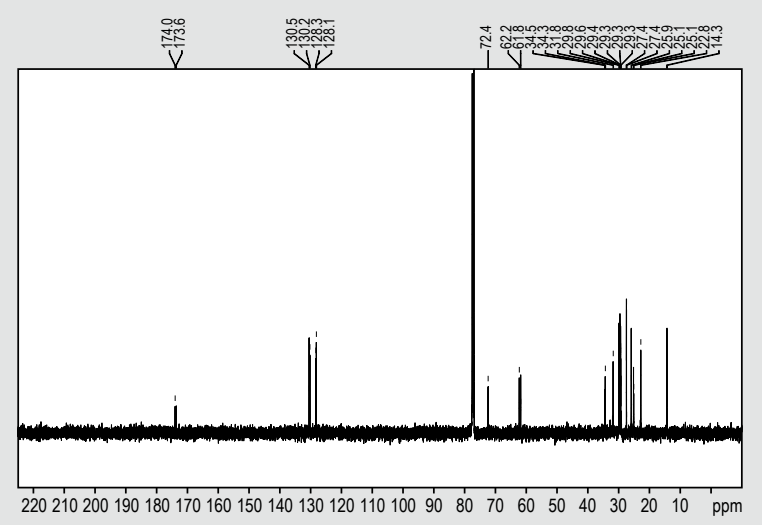

Figure 2. (A) The H-NMR spectra of compound 1 using $\mathrm{CDCl}_{3}$ as the solvent with the spectrometer frequency set at $500 \mathrm{MHz}$. The diagram illustrated 8 different peaks at $\delta_{H} 5.2-5.4, \delta_{H} 4.1-4.2, \delta_{H} 2.7-2.8, \delta_{H} 2.3, \delta_{H} 1.9-2.1, \delta_{H} 1.2-1.3, \delta_{H} 0.96$, and $\delta_{H} 0.87$ respectively. (B) The $\mathrm{C}^{13}$-NMR spectra of compound 1 using $\mathrm{CDCl}_{3}$ as the solvent with the spectrometer frequency set at $125 \mathrm{MHz}$. The spectra showed 4 distinct peaks at $\delta_{C} 174.1, \delta_{C} 128.0-132.2, \delta_{C} 68.6, \delta_{C} 65.3$, and $\delta_{C} 22.8-34.3$ respectively. (C) The H-NMR spectra of Compound 2 using $\mathrm{CDCl}_{3}$ as the solvent with the spectrometer frequency set at $500 \mathrm{MHz}$. The spectra showed 10 peaks at $\delta_{H} 5.2-5.4, \delta_{H} 5.0$ 5.1, $\delta_{H} 4.1-4.4, \delta_{H} 3.7, \delta_{H} 2.7-2.8, \delta_{H} 2.2-2.4, \delta_{H} 1.9-2.1, \delta_{H} 1.5-1.7, \delta_{H} 1.2-1.4$, and $\delta_{H} 0.8-1.0$ respectively. (D) The C-13 NMR spectra of compound 2 using $\mathrm{CDCl}_{3}$ as the solvent with the spectrometer frequency set at $125 \mathrm{MHz}$. The spectra showed 6 distinct peaks at $\delta_{C} 173.6-174.0, \delta_{C} 128.1-130.5, \delta_{C} 68.6, \delta_{C} 72.4, \delta_{C} 61.8-62.2, \delta_{C} 22.8-34.5$, and $\delta_{c} 14.3$ respectively.

Combining the information from both the ${ }^{1} \mathrm{H}-\mathrm{NMR}$ and ${ }^{13} \mathrm{C}$-NMR spectra (Figure $2 \mathrm{~A}$ and $2 \mathrm{~B}$ ), we reasonably deduced that Compound 1 is a 1,3-diglyceride with $\omega-3$, $\omega-6$ or $\omega-9$ unsaturated fatty acids (Alexandri et al., 2017).

The ${ }^{1} \mathrm{H}$-NMR spectrum of Compound 2 was shown in Figure $2 \mathrm{C}$, and the interpretation of the peaks at the same locations are identical to that of Compound 1, whereas peaks located at $\delta_{H} 3.70, \delta_{H} 4.29, \delta_{H} 4.2$ and $\delta_{H} 5.07$ came from the protons connected to a glycerol group. Based on the information, the chemical structure of Compound 2 also resembles that of a triglyceride. The ${ }^{13} \mathrm{C}$-NMR spectrum in Figure 2D indicated that the chemical shifts located at $\delta_{C} 61.8, \delta_{C} 62.2$ and $\delta_{C} 72.4$ with a 1:1:1 ratio splitting pattern could be a carbonyl (C-O) group. The chemical shifts at $\delta_{C} 173.6$ and $\delta_{C} 174.0$ on the other hand, represented the presence of an ester group in the chemical structure.
Combining the information from both the ${ }^{1} \mathrm{H}-\mathrm{NMR}$ and ${ }^{13} \mathrm{C}$-NMR spectra (Figure $2 \mathrm{C}$ and $2 \mathrm{D}$ ), we suspected that Compound 2 is a 1,2-diglyceride with $\omega-3, \omega-6$ or $\omega-9$ unsaturated fatty acids.

\section{LC-MS/MS analysis and statistical analysis of BSFL metabolites}

In our study, the hatched BSFL were fed with sesame throughout the five growth stages. All samples were first processed and analysed using the positive ion mode of LC-MS, and the raw data was subjected to multivariate statistical analyses. First and foremost, we established the PCA model for all five groups: ses, $0 \mathrm{~b}, 2 \mathrm{~b}, 4 \mathrm{~b}$ and $5 \mathrm{~b}$, to get an overview on the relationships between groups (Figure $3 \mathrm{~A})$. Based on the results from Figure 3(A1), the data points from experimental groups $2 \mathrm{~b}, 4 \mathrm{~b}$ and $5 \mathrm{~b}$ were well separated from the controls ( $0 \mathrm{~b}$ and ses). Among three experimental groups, $4 \mathrm{~b}$ and $5 \mathrm{~b}$ were located closest to each other on 

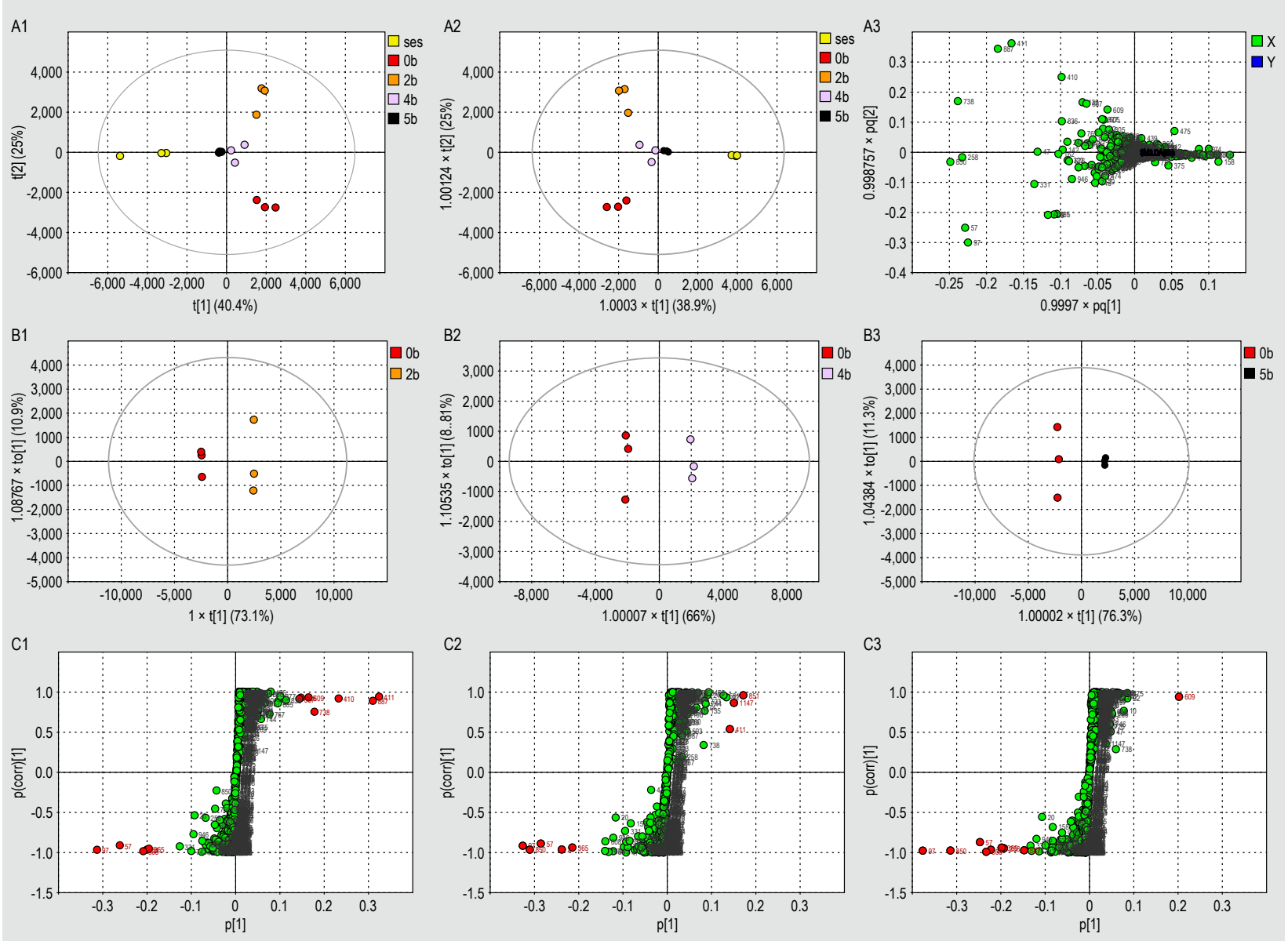

Figure 3. Multivariate statistical analyses of BSFL metabolites using data generated from LC-MS (positive ion mode). (A1) Representative PCA score plot showing different clustering/grouping of samples from the five treatment groups: ses, $0 \mathrm{~b}, 2 \mathrm{~b}$, $4 \mathrm{~b}$ and $5 \mathrm{~b}$. (A2) OPLS-DA model establishing groupings based on differences within groups. (A3) Loading plot illustrating the effect of individual compounds on the OPLS-DA grouping. (B1-B3) OPLS-DA score scatter plots demonstrating the within and between group variation between $0 \mathrm{~b}$ growth control and other growth stages. (C1-C3) Representative OPLS-DA S-plots showing the distribution patterns of compounds contributing to the differences between $0 \mathrm{~b}$ growth control and samples extracted at different growth stages on the score plots.

the graph, representing a closer association between the two and indicating similarities in their metabolite compositions (Figure 3 (A1)). The OPLS-DA model was built subsequently to analyse the intergroup differences. As shown in Figure 3 (A2), the OPLS-DA model captured the small variation between groups compared to the ses control. The effects of metabolite compositions at different developmental stages on the class variation was shown in the OPLS-DA score scatter plot (Figure 3 (A3)).

The three datasets from the $2^{\text {nd }}, 4^{\text {th }}$ and $5^{\text {th }}$ instar were then grouped separately with the stage 0 control $(0 \mathrm{~b}-2 \mathrm{~b}, 0 \mathrm{~b}-4 \mathrm{~b}$, and $0 \mathrm{~b}-5 \mathrm{~b})$ to compare the separation of experimental groups from the growth control. As indicated by Figure 3 (B1-B3), all experimental groups separated well, landing on different side of the axes when individually grouped with $0 \mathrm{~b}$.
Figure 3 (C1-C3) showed the distribution of the components isolated from the BSFL on the S-plots using different group combinations as in Figure 3 (B1-B3). Each point on the $\mathrm{S}$-plot represents a component isolated from the larvae and the potential biomarkers were selected from the list of compounds. These potential biomarkers were then evaluated through high resolution electrospray ionisation mass spectroscopy (HRESIMS) (Figure S2A, S2B) and using Progenesis QI software for identification. As a result, the compounds with molecular weights of 165.079 and 174.111 are the main components causing the differences among the clusters (Table 1 ). Through verifying with the standards, these two compounds were identified as phenylalanine (Phe) and arginine (Arg) respectively.

For the negative ion mode of LC-MS, the controls and experimental groups were analysed with the same method as positive ion mode that described above. Based on the 
Table 1. Chemical properties and relative composition of the potential bioactive metabolites identified from BSFL at different growth stages.

\begin{tabular}{|c|c|c|c|c|c|c|}
\hline \multirow[t]{2}{*}{ Compound name } & \multirow{2}{*}{$\begin{array}{l}\text { Molecular } \\
\text { formula }\end{array}$} & \multirow{2}{*}{$\begin{array}{l}\text { Molecular } \\
\text { weight }\end{array}$} & \multirow{2}{*}{$\begin{array}{l}\text { Retention time } \\
\text { (min) }\end{array}$} & \multicolumn{3}{|c|}{ Relative composition $(\%)^{1}$} \\
\hline & & & & $2 b$ & $4 b$ & $5 b$ \\
\hline Phenylalanine & $\mathrm{C}_{9} \mathrm{H}_{11} \mathrm{NO}_{2}$ & 165.079 & 9.68 & $3.03 \pm 0.35$ & $5.45 \pm 0.54$ & $8.2 \pm 0.22$ \\
\hline Arginine & $\mathrm{C}_{6} \mathrm{H}_{14} \mathrm{~N}_{4} \mathrm{O}_{2}$ & 174.111 & 2.92 & $1.67 \pm 0.24$ & $4.53 \pm 1.53$ & $0.38 \pm 0.18$ \\
\hline Oleic acid & $\mathrm{C}_{18} \mathrm{H}_{34} \mathrm{O}_{2}$ & 282.255 & 32.64 & $33.71 \pm 1.92$ & $51.06 \pm 4.27$ & $35.56 \pm 6.86$ \\
\hline 10-hydroxy-8E-octadecenoic acid & $\mathrm{C}_{18} \mathrm{H}_{34} \mathrm{O}_{3}$ & 298.250 & $28.46 / 31.49$ & $5.18 \pm 0.86$ & $0.80 \pm 0.28$ & $0.31 \pm 0.11$ \\
\hline
\end{tabular}

results of PCA (Figure 4 (A1)), while the data points within experimental groups were dispersed, the clusters of data points from $4 \mathrm{~b}$ and $5 \mathrm{~b}$ remain close to one another and were well separated from the metabolite and growth controls (0b and ses), indicating a close association between the two stages and possible similarities in their metabolite composition. Further evaluation of the internal difference among the groups was done by OPLS-DA model as shown
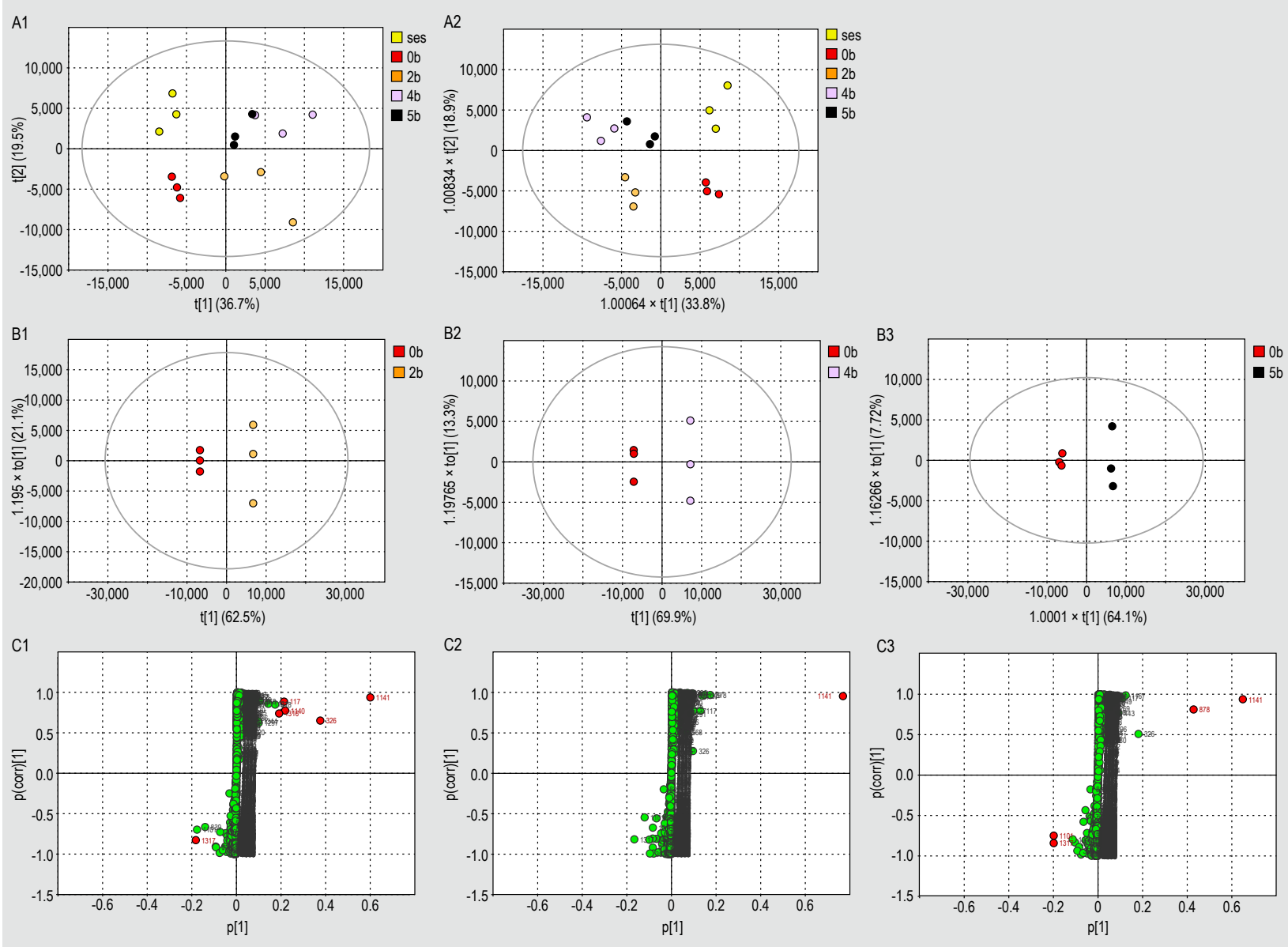

Figure 4. Multivariate statistical analyses of black soldier fly larvae metabolites using data generated from LC-MS (negative ion mode). (A1) Representative PCA score plot showing different clustering/grouping of samples from the five treatment groups: ses, 0b, 2b, 4b and 5b. (A2) OPLS-DA model establishing groupings based on differences within groups. (B1-B3) OPLS-DA score scatter plots demonstrating the within and between group variation between $0 \mathrm{~b}$ growth control and other growth stages. (C1-C3) Representative OPLS-DA S-plots showing the distribution patterns of compounds contributing to the differences between $0 \mathrm{~b}$ growth control and samples extracted at different growth stages on the score plots. 
in Figure 4 (A2). The similarities between $4 \mathrm{~b}$ and $5 \mathrm{~b}$ still remained higher than with other groups in spite of the small variation between groups. As was done in the positive ion mode, the datasets were grouped with $0 \mathrm{~b}$, and all experimental groups separated well when individually compared with the growth control as shown in Figure 4 (B1-B3). Data points on the S-plot (Figure 4 (C1-C3)) represent components isolated from different instar stages of the insects. The differential components selected through the S-plots were metabolites with the molecular weights of 282.255 and 298.250, and were identified to be oleic acid and 10-hydroxy-8E-octadecenoic acid, respectively.

The amount of Phe, Arg, oleic acid and 10-hydroxy-8Eoctadecenoic acid present in the BSFL metabolites at various instars were quantified and indicated in Table 1.

\section{LC-MS/MS and statistical analyses of BSFL faecal metabolites}

Similar to the description in the previous sections, the faecal samples from BSFL from different growth stages were prepared using the same method, except the samples from $0^{\text {th }}$ stage were not taken. All samples were first processed and analysed using the positive ion mode of LC-MS before the multivariate statistical analyses. The four conditions were: sesame residues (ses), faeces from $2^{\text {nd }}(2 \mathrm{~m}), 4^{\text {th }}(4 \mathrm{~m})$ and $5^{\text {th }}$ instar stage $(5 \mathrm{~m})$ of BSFL respectively. As shown in Figure S4A, the OPLS-DA model captured the small variation between groups compared to the ses control, and the groups separated well from one another. Figure S4B showed the distribution of the components isolated from the insect faeces and ses using different grouping methods. The possible biomarkers selected from the S-plots could be mannitol or sorbitol, arabitol or xylitol, and maltose, all paired names represent the stereoisomers with the same molecular formula (Table 2).

The faecal samples from BSFL were analysed using the negative ion mode of LC-MS as well. As shown in Figure S4C, the groups appeared to be well separated from one another according to the OPLS-DA model, and the small variation between groups when compared to the ses control were captured. Figure S4D showed the possible biomarkers selected from the S-plots. Through the negative ion mode analyses of the faecal metabolites in BSFL (Table 2), we found out that the relative content of the compound with the molecular weight of 182.078 , which was supposed to be mannitol or its isomer, showed the same pattern as that in the positive ion mode. Three compounds with the molecular weight of 214.156, 216.099 and 228.084, which were not successfully identified, were all decreased in the $4^{\text {th }}$ stage and increased in the $5^{\text {th }}$ stage. Conversely, the opposite pattern was observed in the compound with the molecular weight of 278.224 which increased during the first three stages, but decreased after reaching the middle stage of development.

\section{Discussion and conclusion}

The black soldier fly is an excellent model organism with a short life cycle that can be easily cultured for research use. Besides the use of BSF in research, it is also an organic fertiliser and a common source of protein for aquaculture,

Table 2. Chemical properties and relative composition of the BSFL faecal metabolites with possible bioactivities identified through LC-MS.

\begin{tabular}{|c|c|c|c|c|c|c|}
\hline \multirow[t]{2}{*}{ Compound name } & \multirow[t]{2}{*}{ Molecular formula } & \multirow[t]{2}{*}{ Molecular weight } & \multirow{2}{*}{$\begin{array}{l}\text { Retention time } \\
\text { (min) }\end{array}$} & \multicolumn{3}{|c|}{ Relative composition (\%) ${ }^{1}$} \\
\hline & & & & $2 m$ & $4 m$ & $5 m$ \\
\hline \multicolumn{7}{|l|}{ Positive ion mode } \\
\hline Arabitol (xylitol) & $\mathrm{C}_{5} \mathrm{H}_{12} \mathrm{O}_{5}$ & 152.068 & 2.84 & $8.94 \pm 1.28$ & $7.62 \pm 1.44$ & $2.11 \pm 0.10$ \\
\hline Unknown & $\mathrm{C}_{10} \mathrm{H}_{21} \mathrm{~N}$ & 155.167 & 9.67 & $4.15 \pm 0.19$ & $7.89 \pm 0.86$ & $6.41 \pm 1.19$ \\
\hline Unknown & $\mathrm{C}_{9} \mathrm{H}_{19} \mathrm{NO}$ & 157.146 & 9.79 & $6.44 \pm 0.24$ & $4.15 \pm 1.68$ & $4.38 \pm 0.47$ \\
\hline Mannitol (sorbitol) & $\mathrm{C}_{6} \mathrm{H}_{14} \mathrm{O}_{6}$ & 182.079 & 2.80 & $0.07 \pm 0.00$ & $0.10 \pm 0.00$ & $0.71 \pm 0.01$ \\
\hline Maltose & $\mathrm{C}_{12} \mathrm{H}_{22} \mathrm{O}_{11}$ & 342.116 & 2.99 & $0.68 \pm 0.01$ & $1.18 \pm 0.51$ & $3.86 \pm 0.25$ \\
\hline \multicolumn{7}{|l|}{ Negative ion mode } \\
\hline Mannitol (sorbitol) & $\mathrm{C}_{6} \mathrm{H}_{14} \mathrm{O}_{6}$ & 182.078 & 2.78 & $0.32 \pm 0.28$ & $0.85 \pm 0.50$ & $8.43 \pm 0.61$ \\
\hline Unknown & $\mathrm{C}_{12} \mathrm{H}_{22} \mathrm{O}_{3}$ & 214.156 & 17.7 & $1.36 \pm 0.19$ & $0.90 \pm 0.05$ & $1.38 \pm 1.05$ \\
\hline Unknown & $\mathrm{C}_{10} \mathrm{H}_{16} \mathrm{O}_{5}$ & 216.099 & 10.2 & $3.23 \pm 0.59$ & $0.89 \pm 1.15$ & $3.26 \pm 0.74$ \\
\hline Unknown & $\mathrm{C}_{7} \mathrm{H}_{16} \mathrm{O}_{8}$ & 228.084 & 2.80 & $0.33 \pm 0.01$ & $0.29 \pm 0.18$ & $2.05 \pm 0.41$ \\
\hline a-linolenic acid & $\mathrm{C}_{18} \mathrm{H}_{30} \mathrm{O}_{2}$ & 278.224 & 32.63 & $2.29 \pm 1.45$ & $5.42 \pm 3.66$ & $0.89 \pm 0.42$ \\
\hline
\end{tabular}

\footnotetext{
${ }^{1}$ The relative composition was calculated by dividing the target peak area by the total peak area (mean \pm SD).
} 
animal feed, pet food and human nutrition. Natural biologically active substances can be extracted from plants, animals and microorganisms. There are discoveries in recent years suggesting benefits in certain insects: not only are they an excellent source of protein and lipid, they are highly efficient waste bio-converters (El-Hack et al., 2020). As opposed to traditional feed, they barely emit greenhouse gases and consume water. One of the most beneficial is $H$. illucens larvae, which has high nutritional value and a variety of enzymes convert organic waste in their digestive tract (Almeida et al., 2020). BSFL is able to perform significant biological functions such as reducing pathogenic Salmonella spp. in a waste management system (Lalander et al., 2013). Antimicrobial peptides found in BSFL would help to reduce the microbial load in larvae used as animal feed without additional costs (Vogel et al., 2018). The bioactive compounds present in BSFL like fatty acids, amino acids and enzymes suggests their potential in the development of cosmetics and pharmaceutical products (Almeida et al., 2020). In spite of all of the functions possessed in BSFL, the studies of metabolomics on BSFL remains limited.

Sesame is a popular home-cooked seasoning in Asia. After mechanically processed for oil extraction, the sesame residues possess high protein and lipid content and variable nutrients. The most common application of sesame residues is compost with carbon compounds to fertilise the land, feedstuff for poultry, swine, and fish. Sesame cake as supplementation on feed can increase body weight gain and enhance carcass parameters of sheep (Fitwi and Tadesse, 2013), increase daily gain and feed conversion efficiency of Awassi lambs (Omar, 2002), and increase growth and feed conversion ratio of carp (Mazid et al., 1997). Nevertheless, when it is used as poultry feed, there is a growth restriction when feed ingredient is substituted with sesame residues by $30 \%$ (Xu et al., 2014). To increase its availability, some fermentation processes prior feed might be needed for better digestion and absorption. In our study, we make use of sesame residues as feed for BSFL rearing.

In this paper, the metabolites of BSFL using sesame residues, the food industrial waste, as the energy source were first analysed and reported. The larvae were reared for 92 days until sacrifice. The growth period of larvae was reported to be 15-36.7 days (Kim et al., 2021). Considering the size of larvae, the body length was about $1.8 \mathrm{~cm}$ at the $5^{\text {th }}$ stage, which was smaller than had been reported (Tran et al., 2015). Diet-reared BSFL was reported to have reduced size comparison to the wild (Tomberlin, 2003), and several factors on substrates and rearing conditions could affect the growth of BSFL including temperature, food supply and composition (Kim et al., 2021). The ratio of larvae number to gram of sesame residues were 9:1 to 2.5:1, which was higher than 2:1 suggested in the literature, and might be the possible factor (Kim et al., 2021). For the ethanolic extraction method, Kenari et al. reported that the highest amount of total phenolic compounds is observed in ethanol-ultrasonic extract of sesame (Esmaeilzadeh Kenari et al., 2014). By using HPLC-DAD and HPLC-MS combining with the PCA and OPLS-DA analysis, several differential components of $S$. indium and metabolites from different growth stages of BSFL have been discovered and identified. In S. indium, the main components utilised by BSFL for metabolism has been purified and identified as diacylglycerol (DAG). Literature showed that acylglycerols are prevalent dietary lipids consumed by insects (Holtof et al., 2019). Furthermore, DAG was reported to be the major transport lipid shuttle in the BSFL (Leong and Kutty, 2020).

According to LC-MS/MS with ESI positive ion mode analyses of the BSFL metabolites, the identified compounds, Phe and Arg, are essential amino acids that serve as building blocks for proteins in insects (Lalander et al., 2019). Osanai et al. observed changes in amounts of Phe and Arg during the growth of silkmoth: Phe changed irregularly with age, while Arg increased in middle age and decreased gradually after (Osanai and Yonezawa, 1984). Besides, the accumulation of essential amino acids is probably due to essential amino acid imbalance, which affects lifespan of insects (Grandison et al., 2009). Sesame cake was reported to contain all of the essential amino acids (Yang et al., 2021). However, the composition might not be well-balanced for BSFL, the resulting dietary restriction could be the possible reason for the long lifespan of BSFL in this study. Phe is the initial precursor for aromatic compounds such as tyrosine, melanin, and dopamine (Brunet, 1980). Among the three aromatic compounds listed, melanin formation is responsible for cuticular coloration and sclerotisation occurring during metamorphosis (Chen et al., 2013). Additionally, Phe metabolism is associated with several other physiological processes including neuromodulation and immune responses (Simonet et al., 2016). Other than the nutritional requirement of Arg, the conversion of Arg into proline in insects plays a key role in flight muscle metabolism of winged adult (Reddy and Campbell, 1969). The relative content of these two amino acids gradually increases as the larvae mature except for the percentage of Arg in 5b larvae, and the underlying reason requires further investigation.

For the ESI negative ion mode analysis of BSFL metabolites, metabolites with the molecular weights of 282.255 and 298.250 were the main components contributing to the differences amongst the clusters (Table 1). Through cross comparison with the standards by using HRESIMS analysis (Figure S3), molecular weight of 282.255 was found to be oleic acid (Table 1). Literature has shown that the amount of oleic acid in the cell membrane is responsible for membrane fluidity, and the percentage content in the cell membrane is upregulated during low-temperature (Michaud and Denlinger, 2006). The fatty acid composition 
and the relative contents of sesame residue calculated from the LC-MS spectrum are oleic acid, linoleic acid, linolenic acid, palmitic acid, stearic acid, and arachidic acid were $17.29 \pm 1.10,63.41 \pm 3.80,0.50 \pm 0.07,9.83 \pm 0.15,8.92 \pm 2.44$ and $0.06 \pm 0.05 \%$, respectively. Since the $S$. indicum residue is composed of approximately $20 \%$ oleic acids, the diet contributes to a portion of the oleic acid found in the metabolites of BSFL. The other portion of the oleic acid found in the metabolites can be attributed to the indirect conversion of other components found in sesame. Among all growth stages, the $4 \mathrm{~b}$ larvae had the highest relative content of oleic acid, representing the highest cumulative metabolic rate of this compound. Interestingly, another compound with the molecular weight of 298.250 showed a fragment ion of $155.11 \mathrm{~m} / \mathrm{z}$ in the MS2 spectrum, and was deduced to be 10-hydroxy-8E-octadecenoic acid, an oxidised form of oleic acid with an extra oxygen atom (Martin-Arjol et al., 2010). Unlike the compounds previously discussed, its relative content decreases over time as the larvae matured, signifying the decrease in metabolism of the compound. Pseudomonas aeruginosa (PR3) had been reported to produce 10-hydroxy-8-octadecenoic acid from oleic acid (Kim et al., 2000). And previous study had demonstrated that Pseudomonas is a conserved member of BSFL gut microbiota raised in different substrates (Tanga et al., 2021). This may help explain the effects of insect microbiota on lipid metabolism although the effect of insect metabolism itself to oleic acid is nonnegligible.

The predicted faecal metabolites were obtained through the same analytical method as described previously. According to the result of positive ion mode shown in Table 2, the relative content of compound with the molecular weight of 182.079 , which was deduced to be mannitol or its stereoisomer, increased proportionally in the faeces as the consumption of sesame increased in the larvae along with the growth stages. Literature has shown that sorbitol and mannitol accumulation provides a mechanism for thermo- and osmo-protection in insects, which could be the explanation for their accumulation within the insect body (Hendrix and Salvucci, 1998). Similarly, arabitol was found to serve similar functions in thermoregulation compare to the polyhydric alcohols stated previously. With its percentage decreasing in the faecal metabolite as the larvae develop, it is possible that the compounds are retained within the larvae for homeostasis (Colinet et al., 2016). The possibility of these compounds being associated with the metabolism of gut bacteria is positive. The insect gut bacteria can convert cellulose to monosaccharide (Jing et al., 2020). Kim et al isolated several gut bacteria from BSFL, and found that these bacteria use mannitol, arabitol, sorbitol, and xylitol as nutrient (Kim et al., 2014). The compound with molecular weight of 342.116 was deduced to be maltose, which is a common source of carbohydrates of insects, and its proportion gradually increased along with the growth stages. This accumulation of maltose may be due to the inhibition of maltase by pinoresinol in sesame seeds (Wikul et al., 2012). Two metabolites with the molecular weight of 155.167 and 157.146 were not identified, of which content was relatively high in the respective $4^{\text {th }}$ and $2^{\text {nd }}$ growth stage.

As for the negative ion mode analyses on faecal samples, according to the literature on main fatty acids found in $S$. indium and BSFL feeds, we speculate that the unknown compound with the molecular weight of 278.224 is $\alpha$-linolenic acid (ALA, 18:3, $\omega-3$ ), which is essential for the normal development and metamorphosis in most insects (Dadd, 1981; Lalander et al., 2019). Insects generally obtain fat through their diet and break them into fatty acids by lipase and absorbed through the intestine. The fatty acids are then converted into DAG to partake in membrane synthesis, transport and storage of metabolites and cellular signal transduction. Summarising the experimental results, the relative ratio of ALA and oleic acid found in the larvae and faeces was highest in the $4^{\text {th }}$ growth stage and decreased before prepupal stage, indicating higher energy consumption at later growth stages of BSFL.

Processing high protein and lipid content, the sesame residues is a suitable feed to be incorporated in BSFL's meal. We anticipate that sesame residues can be applied in contexts including, but not limited to agriculture, environment, and food industry to improve insect rearing on a large scale. Among the larvae and faeces of different growth stages of BSFL, the results showed that the main components contributing to the differences among the clusters were Phe, Arg, ALA, oleic acid, 10-hydroxy8E-octadecenoic acid, maltose, mannitol (or sorbitol) and arabitol (or xylitol). Relative changes in the amount of these biomarkers during different instar stages may be associated with varied nutritional requirements and energy consumption of BSFL at each growth period. With larvae having different nutritional compositions at different instar stages, larvae can be harvested at different growth stages to provide certain nutrients and better suit the nutritional requirements of different populations. For instance, the rich Arg content in various instars can serve as dietary supplementation for improving reproductive, cardiovascular, pulmonary, renal, gastrointestinal, liver and immune functions, as well as facilitating wound healing, enhancing insulin sensitivity, and maintaining tissue integrity (Wu et al., 2009). Similarly, consumption of ALA and oleic acid-rich instars can reduce cardiovascular disease risk, serve as potential nutraceutical to protect the brain from stroke, characterised by its pleiotropic effects in neuroprotection, vasodilation of brain arteries, and neuroplasticity (Blondeau et al., 2015). The present study revealed a part of metabolic changes important in the BSFL lifecycle, these findings could be a clue for further studies focusing on metabolism of BSFL and its gut microbiota. Since the amounts of these nutritional compounds differ in 
various growth stages, this information could potentially be used to obtain optimised nutrient composition or shorten the harvest time period when using BSFL as feed.

\section{Supplementary material}

Supplementary material can be found online at https://doi. org/10.3920/JIFF2021.0045.

Table S1. Mobile phase composition of the HPLC gradient used for analysing black soldier fly larvae metabolites.

Table S2. Optimised electrospray ionisation mass spectroscopy (ESI-MS) parameters for analysing black soldier fly larvae metabolites.

Figure S1. Extraction of compound 1 and 2 from sesame residue illustrated in a flow chart.

Figure S2. HRESI-MS/MS spectrum of (A) phenylalanine $(\mathrm{M} . \mathrm{W} .=165)$ and (B) arginine (M.W.=174).

Figure S3. HRESI-MS/MS spectra of $\alpha$-linolenic acid (M.W.=278).

Figure S4. Multivariate statistical analyses of BSFL faecal metabolites using data generated from LC-MS (positive ion mode).

Figure S5. Multivariate statistical analyses of BSFL faecal metabolites using data generated from LC-MS (negative ion mode).

\section{Acknowledgements}

We thank you to Dr YueSea Eric Chen for his early suggestions and discussions on the physiological functions of Black Soldier Fly. We thank Raycome International Corp. for providing $H$. illucens $\mathrm{L}$. and the feed of sesame residue. We thank Taipei Medical University and National Taiwan University Precious Instrument Center for providing NMR and UHPLC-MS systems. This research was supported by a grant from the Ministry of Science and Technology of the Republic of China (Taiwan) (MOST 107-2320-B-038019-MY3).

\section{Conflict of interest}

The authors declare no conflict of interest.

\section{References}

Akihisa, T., Yasukawa, K., Yamaura, M., Ukiya, M., Kimura, Y., Shimizu, N. and Arai, K., 2000. Triterpene alcohol and sterol ferulates from rice bran and their anti-inflammatory effects. Journal of Agricultural and Food Chemistry 48: 2313-2319.
Alexandratos, N. and Bruinsma, J., 2012. World agriculture towards 2030/2050: the 2012 revision. FAO, Rome, Italy.

Alexandri, E., Ahmed, R., Siddiqui, H., Choudhary, M.I., Tsiafoulis, C.G. and Gerothanassis, I.P., 2017. High resolution NMR spectroscopy as a structural and analytical tool for unsaturated lipids in solution. Molecules 22: 1663.

Almeida, C., Rijo, P. and Rosado, C., 2020. Bioactive compounds from Hermetia Illucens larvae as natural ingredients for cosmetic application. Biomolecules 10: 976.

Blondeau, N., Lipsky, R.H., Bourourou, M., Duncan, M.W., Gorelick, P.B. and Marini, A.M., 2015. Alpha-linolenic acid: an omega-3 fatty acid with neuroprotective properties - ready for use in the stroke clinic? BioMed Research International 2015: 519830.

Brunet, P.C., 1980. The metabolism of the aromatic amino acids concerned in the cross-linking of insect cuticle. Insect Biochemistry 10: 467-500

Chen, P., Li, L., Wang, J., Li, H., Li, Y., Lv, Y. and Lu, C., 2013. BmPAH catalyzes the initial melanin biosynthetic step in Bombyx mori. PLoS ONE 8: e71984.

Churchward-Venne, T.A., Pinckaers, P.J., Van Loon, J.J. and Van Loon, L.J., 2017. Consideration of insects as a source of dietary protein for human consumption. Nutrition Reviews 75: 1035-1045.

Colinet, H., Renault, D., Javal, M., Berková, P., Šimek, P. and Koštál, V., 2016. Uncovering the benefits of fluctuating thermal regimes on cold tolerance of drosophila flies by combined metabolomic and lipidomic approach. Biochimica et Biophysica Acta - Molecular and Cell Biology of Lipids 1861: 1736-1745.

Dadd, R., 1981. Essential fatty acids for mosquitoes, other insects and vertebrates. In: Bhaskaran, G., Friedman S. and Rodriguez, J.G. (eds.) Current topics in insect endocrinology and nutrition. Springer, Boston, MA, USA, pp. 189-214.

De Smet, J., Wynants, E., Cos, P. and Van Campenhout, L., 2018. Microbial community dynamics during rearing of black soldier fly larvae (Hermetia illucens) and impact on exploitation potential. Applied and Environmental Microbiology 84: e02722-17.

El-Hack, A., Mohamed, E., Shafi, M.E., Alghamdi, W.Y., Abdelnour, S.A., Shehata, A.M., Noreldin, A.E., Ashour, E.A., Swelum, A.A. and Al-Sagan, A.A., 2020. Black soldier fly (Hermetia illucens) meal as a promising feed ingredient for poultry: a comprehensive review. Agriculture 10: 339.

Elhag, O., Zhou, D., Song, Q., Soomro, A.A., Cai, M., Zheng, L., Yu, Z. and Zhang, J., 2017. Screening, expression, purification and functional characterization of novel antimicrobial peptide genes from Hermetia illucens (L.). PLoS ONE 12: e0169582.

Esmaeilzadeh Kenari, R., Mohsenzadeh, F. and Amiri, Z.R., 2014. Antioxidant activity and total phenolic compounds of Dezful sesame cake extracts obtained by classical and ultrasound-assisted extraction methods. Food Science \& Nutrition 2: 426-435.

Fitwi, M. and Tadesse, G., 2013. Effect of sesame cake supplementation on feed intake, body weight gain, feed conversion efficiency and carcass parameters in the ration of sheep fed on wheat bran and teff (Eragrostis teff) straw. Momona Ethiopian Journal of Science 5: 89-106.

Grandison, R.C., Piper, M.D. and Partridge, L., 2009. Amino-acid imbalance explains extension of lifespan by dietary restriction in Drosophila. Nature 462: 1061-1064. 
Hall, R.D. and Gerhardt, R.R., 2002. Flies (Diptera). In: Mullen, G.R. and Durden, L.A. (eds.) Medical and veterinary entomology. Academic Press, San Diego, CA, USA, pp. 127-145.

Hendrix, D.L. and Salvucci, M.E., 1998. Polyol metabolism in homopterans at high temperatures: accumulation of mannitol in aphids (Aphididae: Homoptera) and sorbitol in whiteflies (Aleyrodidae: Homoptera). Comparative Biochemistry and Physiology Part A: Molecular \& Integrative Physiology 120: 487-494.

Holtof, M., Lenaerts, C., Cullen, D. and Broeck, J.V., 2019. Extracellular nutrient digestion and absorption in the insect gut. Cell and Tissue Research 377: 397-414.

Huang, C., Feng, W., Xiong, J., Wang, T., Wang, W., Wang, C. and Yang, F., 2019. Impact of drying method on the nutritional value of the edible insect protein from black soldier fly (Hermetia illucens L.) larvae: amino acid composition, nutritional value evaluation, in vitro digestibility, and thermal properties. European Food Research and Technology 245: 11-21.

Jiang, C.L., Jin, W.Z., Tao, X.H., Zhang, Q., Zhu, J., Feng, S.Y., Xu, X.H., Li, H.Y., Wang, Z.H. and Zhang, Z.J., 2019. Black soldier fly larvae (Hermetia illucens) strengthen the metabolic function of food waste biodegradation by gut microbiome. Microbial Biotechnology 12: 528-543.

Jing, T.-Z., Qi, F.-H. and Wang, Z.-Y., 2020. Most dominant roles of insect gut bacteria: digestion, detoxification, or essential nutrient provision? Microbiome 8: 38.

Kim, C.-H., Ryu, J., Lee, J., Ko, K., Lee, J.-Y., Park, K.Y. and Chung, H., 2021. Use of black soldier fly larvae for food waste treatment and energy production in Asian countries: a review. Processes 9: 161.

Kim, E., Park, J., Lee, S. and Kim, Y., 2014. Identification and physiological characters of intestinal bacteria of the black soldier fly, Hermetia illucens. Korean Journal of Applied Entomology 53: 15-26.

Kim, H., Gardner, H.W. and Hou, C.T., 2000. 10 (S)-hydroxy-8 (E)octadecenoic acid, an intermediate in the conversion of oleic acid to 7, 10-dihydroxy-8 (E)-octadecenoic acid. Journal of the American Oil Chemists' Society 77: 95-99.

Lalander, C., Diener, S., Magri, M.E., Zurbrügg, C., Lindström, A. and Vinnerås, B., 2013. Faecal sludge management with the larvae of the black soldier fly (Hermetia illucens) - from a hygiene aspect. Science of the Total Environment 458: 312-318.

Lalander, C., Diener, S., Zurbrügg, C. and Vinnerås, B., 2019. Effects of feedstock on larval development and process efficiency in waste treatment with black soldier fly (Hermetia illucens). Journal of Cleaner Production 208: 211-219.

Leong, S.Y. and Kutty, S., 2020. Characteristic of Hermetia illucens fatty acid and that of the fatty acid methyl ester synthesize based on upcycling of perishable waste. Waste and Biomass Valorization 11: 5607-5614.

Müller, A., Wolf, D. and Gutzeit, H.O., 2017. The black soldier fly, Hermetia illucens - a promising source for sustainable production of proteins, lipids and bioactive substances. Zeitschrift für Naturforschung C 72: 351-363.

Martin-Arjol, I., Bassas-Galia, M., Bermudo, E., Garcia, F. and Manresa, A., 2010. Identification of oxylipins with antifungal activity by LCMS/MS from the supernatant of Pseudomonas 42A2. Chemistry and Physics of Lipids 163: 341-346.
Mazid, M., Zaher, M., Begum, N., Ali, M. and Nahar, F., 1997. Formulation of cost-effective feeds from locally available ingredients for carp polyculture system for increased production. Aquaculture 151: 71-78.

McKee, J.K., 2003. Sparing nature: the conflict between human population growth and earth's biodiversity. Rutgers University Press, New Brunswick, NJ, USA.

Michaud, M.R. and Denlinger, D.L., 2006. Oleic acid is elevated in cell membranes during rapid cold-hardening and pupal diapause in the flesh fly, Sarcophaga crassipalpis. Journal of Insect Physiology 52: 1073-1082.

Miraj, S. and Kiani, S., 2016. Bioactivity of Sesamum indicum: a review study. Der Pharmacia Lettre 8: 328-334.

Mwangi, M.N., Oonincx, D.G., Stouten, T., Veenenbos, M., MelseBoonstra, A., Dicke, M. and Van Loon, J.J., 2018. Insects as sources of iron and zinc in human nutrition. Nutrition Research Reviews 31: 248-255.

Newton, L., Sheppard, C., Watson, D.W., Burtle, G. and Dove, R., 2005. Using the black soldier fly, Hermetia illucens, as a value-added tool for the management of swine manure. Animal and Poultry Waste Management Center, North Carolina State University, Raleigh, NC, USA.

Omar, J.A., 2002. Effects of feeding different levels of sesame oil cake on performance and digestibility of Awassi lambs. Small Ruminant Research 46: 187-190.

Osanai, M. and Yonezawa, Y., 1984. Age-related changes in amino acid pool sizes in the adult silkmoth, bombyx mori, reared at low and high temperature; a biochemical examination of the rate-of-living theory and urea accumulation when reared at high temperature. Experimental Gerontology 19: 37-51.

Park, S.-I., Kim, J.-W. and Yoe, S.M., 2015. Purification and characterization of a novel antibacterial peptide from black soldier fly (Hermetia illucens) larvae. Developmental \& Comparative Immunology 52: 98-106.

Reddy, S.R.R. and Campbell, J.W., 1969. Arginine metabolism in insects. Role of arginase in proline formation during silkmoth development. Biochemical Journal 115: 495-503.

Simonet, P., Gaget, K., Parisot, N., Duport, G., Rey, M., Febvay, G., Charles, H., Callaerts, P., Colella, S. and Calevro, F., 2016. Disruption of phenylalanine hydroxylase reduces adult lifespan and fecundity, and impairs embryonic development in parthenogenetic pea aphids. Scientific Reports 6: 34321.

Spranghers, T., Ottoboni, M., Klootwijk, C., Ovyn, A., Deboosere, S., De Meulenaer, B., Michiels, J., Eeckhout, M., De Clercq, P. and De Smet, S., 2017. Nutritional composition of black soldier fly (Hermetia illucens) prepupae reared on different organic waste substrates. Journal of the Science of Food and Agriculture 97: 2594-2600.

Tanga, C.M., Waweru, J.W., Tola, Y.H., Onyoni, A.A., Khamis, F.M., Ekesi, S. and Paredes, J.C., 2021. Organic waste substrates induce important shifts in gut microbiota of black soldier fly (Hermetia illucens L.): coexistence of conserved, variable, and potential pathogenic microbes. Frontiers in Microbiology 12: 635881.

Tomberlin, J.K., 2003. Biological, behavioral, and toxicological studies on the black soldier fly (Diptera: Stratiomyidae). PhD-thesis, University of Georgia, Athens, GA, USA. 
Tran, G., Gnaedinger, C. and Mélin, C., 2015. Black soldier fly larvae (Hermetia illucens). Available at: https://www.feedipedia.org/ node/16388

Van Huis, A., Van Itterbeeck, J., Klunder, H., Mertens, E., Halloran, A., Muir, G. and Vantomme, P., 2013. Edible insects: future prospects for food and feed security. FAO, Rome, Italy.

Vogel, H., Müller, A., Heckel, D.G., Gutzeit, H. and Vilcinskas, A., 2018. Nutritional immunology: diversification and diet-dependent expression of antimicrobial peptides in the black soldier fly Hermetia illucens. Developmental \& Comparative Immunology 78: 141-148.

Wang, Y.-S. and Shelomi, M., 2017. Review of black soldier fly (Hermetia illucens) as animal feed and human food. Foods 6: 91.

Wikul, A., Damsud, T., Kataoka, K. and Phuwapraisirisan, P., 2012. (+)-Pinoresinol is a putative hypoglycemic agent in defatted sesame (Sesamum indicum) seeds though inhibiting $\alpha$-glucosidase. Bioorganic \& Medicinal Chemistry Letters 22: 5215-5217.
Wu, G., Bazer, F.W., Davis, T.A., Kim, S.W., Li, P., Rhoads, J.M., Satterfield, M.C., Smith, S.B., Spencer, T.E. and Yin, Y., 2009. Arginine metabolism and nutrition in growth, health and disease. Amino Acids 37: 153-168.

Xu, W.-J., Zhang, Y., Ji, Y.-S., Yang, T., Yang, C. and Gan, C.-J., 2014. Status and Recent investigations of utilizing sesame seed meal. Academic Periodical of Farm Products Processing 6: 64-67.

Yang, K., Xu, T.-R., Fu, Y.-H., Cai, M., Xia, Q.-L., Guan, R.-F., Zou, X.-G. and Sun, P.-L., 2021. Effects of ultrasonic pre-treatment on physicochemical properties of proteins extracted from cold-pressed sesame cake. Food Research International 139: 109907.

Zhang, X., Yang, M., Song, F., Zhang, H. and Feng, F., 2013. Antimicrobial activity of selected fatty acids and their derivatives. Journal of Zhejiang University (Agriculture and Life Sciences) 39: $155-160$ 
